\title{
The Superconducting Magnets of the ILC Beam Delivery System ${ }^{* \dagger}$
}

\author{
B. Parker, M. Anerella, J. Escallier, P. He, A. Jain, A. Marone \\ BNL, Upton, NY 11973, USA \\ Y. Nosochkov, A. Seryi \\ Stanford Linear Accelerator Center, 2575 Sand Hill Road, Menlo Park, CA 94025, USA
}

\begin{abstract}
The ILC Beam Delivery System (BDS) uses a variety of superconducting magnets to maximize luminosity and minimize background. Compact final focus quadrupoles with multifunction correction coils focus incoming beams to few nanometer spot sizes while focusing outgoing disrupted beams into a separate extraction beam line. Anti-solenoids mitigate effects from overlapping focusing and the detector solenoid field. Far from the interaction point (IP) strong octupoles help minimize IP backgrounds. A low-field but very large aperture dipole is integrated with the detector solenoid to reduce backgrounds from beamstrahlung pairs generated at the IP. Physics requirements and magnetic design solutions for the BDS superconducting magnets are reviewed in this paper.
\end{abstract}

Presented at the 2007 Particle Accelerator Conference (PAC 07)

Albuquerque, New Mexico, USA, 25-29 June, 2007

\footnotetext{
* This manuscript has been authored by Brookhaven Science Associates, LLC under Contract No. DE-AC02-98CH1-886 with U.S. Department of Energy.

${ }^{\dagger}$ This work was supported by Department of Energy contract DE-AC02-76SF00515.
} 


\title{
THE SUPERCONDUCTING MAGNETS OF THE ILC BEAM DELIVERY SYSTEM*
}

\author{
B. Parker", M. Anerella, J. Escallier, P. He, A. Jain, A. Marone, BNL, Upton, NY, 11973, U.S.A. \\ Y. Nosochkov and A. Seryi, SLAC, Menlo Park, CA 94025, U.S.A.
}

\begin{abstract}
The ILC Beam Delivery System (BDS) uses a variety of superconducting magnets to maximize luminosity and minimize background. Compact final focus quadrupoles with multifunction correction coils focus incoming beams to few nanometer spot sizes while focusing outgoing disrupted beams into a separate extraction beam line. Anti-solenoids mitigate effects from overlapping focusing and the detector solenoid field. Far from the interaction point (IP) strong octupoles help minimize IP backgrounds. A low-field but very large aperture dipole is integrated with the detector solenoid to reduce backgrounds from beamstrahlung pairs generated at the IP. Physics requirements and magnetic design solutions for the BDS superconducting magnets are reviewed in this paper.
\end{abstract}

\section{COMPACT IR MAGNETS FOR 14 MR}

In the ILC $14 \mathrm{mr}$ crossing angle layout incoming and extraction beams are focused in independently. With a distance (denoted $\mathrm{L}^{*}$ ) of the first magnet, QD0, to the IP of $3.5 \mathrm{~m}$, the beam separation at QD0 is $49 \mathrm{~mm}$. This small separation is accommodated via compact coil windings produced using BNL's direct wind technology [1]; however, even with compact coils care is taken so that external field does not impact the extracted beam passing just outside QD0.

We reduce QD0's external field with a weak active shield coil of opposite polarity that runs in series with the main coil windings. With the shield energized, magnetic flux passes between the inner and outer coil structure rather than spreading outside the coil package. The shield coil reduces the gradient inside QD0 but this impact is minimized by keeping the inner coil as radially compact and pushing the shield coil radius out as far as the crossing angle geometry allows. A shielded prototype coil, that fit in an existing small dewar was wound and successfully tested [2]. The actual QD0 coil design has extra space between the main and shield coils for He-II cooling and trim current taps for shield fine tuning.

For optics flexibility the QD0 coil pack has dipole, skew-dipole and skew-quadrupole correction windings. Next to QD0 is another coil package with octupole, sextupole, skew-sextupole, dipole and skew-dipole windings. These correction coils have negligible impact at the extraction line and are not actively shielded.

\footnotetext{
"This manuscript has been authored by Brookhaven Science Associates, LLC under Contract No. DE-AC02-98CH1-886 with U.S. Department of Energy. The United States Government retains, and the publisher, by accepting article for publication, acknowledges, a world-wide license to publish or reproduce published form of this manuscript, or allow others to do so, for the United states Government purposes. \#parker@bnl.gov
}

Alongside the incoming beam line is the first extraction line quadrupole, QDEX1, which is similar to QD0, with active shielding and correction coils. But QDEX1 has a larger clear aperture to minimize energy deposition from the outgoing beam. At present three QD0 L*s, 3.5, 4.0 and $4.5 \mathrm{~m}$, are studied and different QDEX1 designs exist for three different extraction line starting points [3].

Further from the IP there is a second set of magnets, the QF1 grouping, with properties similar to those of the QD0 grouping. The most significant difference is that QF1 is well outside the detector solenoid and has sufficient transverse separation from the extraction beam, for it to have a magnetic yoke for passive external field shielding and thus no active shield coil is needed. QF1 has its own associated sextupole, octupole etc. coil package and neighboring extraction line quadrupole QFEX1.

The QD0 and QF1 magnet groupings are housed in two independent cryostats in order to facilitate the push-pull scenario where two experiments take turns sharing beam time at a single IP. Swapping detectors at a single IP saves considerable project cost for conventional facilities and requires fewer beam line technical components, but adds other complications. In order to make the changeover as rapid as possible the IR magnets and experimental detector are to be kept cold during a switchover. The experiments have QD0 magnet groupings in cryostats that move with the detectors but they share a fixed QF1 magnet grouping that remains in place. Between QD0 and QF1 is warm beam pipe with vacuum valves and pump out ports to make natural break points.

\section{FORCE NEUTRAL ANTI-SOLENOID}

In addition to the magnets described so far, the ILC IR optics uses anti-solenoids to locally correct deleterious optics effects due to QD0 focusing overlapping the detector solenoidal field [4]. If left uncompensated, this overlap leads to an effective beam size increase at the IP and reduces luminosity. To be effective the anti-solenoid does not have to completely cancel the detector field overlapping QD0; in fact it is shorter than QD0 and only modifies the field profile near one end.

A simple anti-solenoid coil would experience a strong, multi-ton, repulsion from the main detector solenoid and is not suitable for integration into the QD0 cryostat. But integrating a large anti-solenoid coil into each detector is also quite challenging. Instead we use a force neutral antisolenoid scheme. The repulsive force experienced by an isolated anti-solenoid coil is related both to the field it produces on axis and its cross sectional area (aperture). It is possible to arrange to have two overlapping coils of opposite polarity but different areas in a way that their 


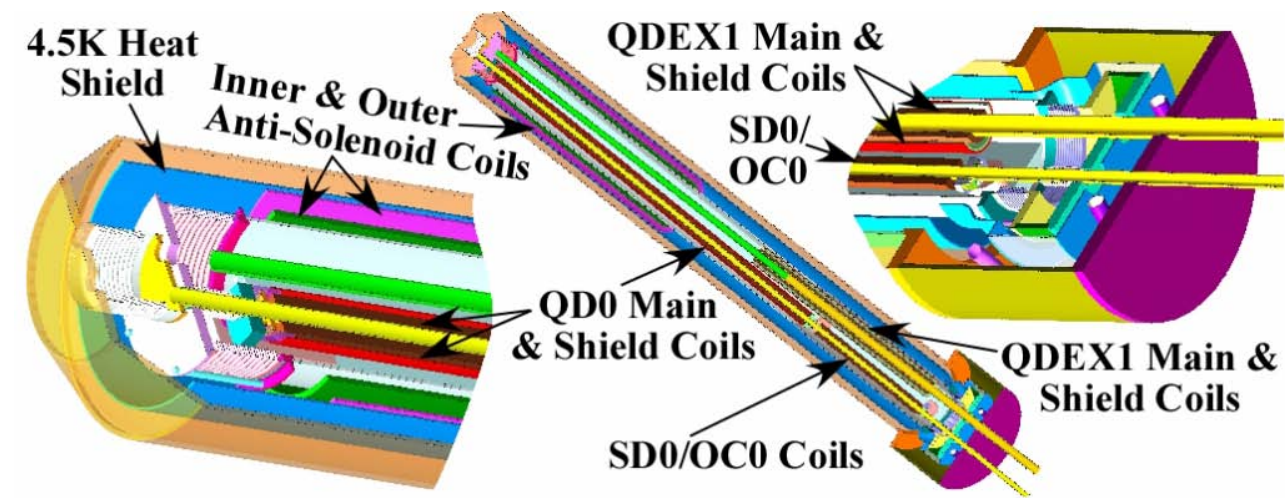

Figure 1. The QD0 Magnet Grouping shown with a Force Neutral Anti-Solenoid in a QD0 Common Cryostat.

repulsive forces balance but maintain a non-zero antisolenoid field inside, as shown in Fig. 1.

A bonus with our force neutral coil configuration is that much like the active shielding discussed earlier, the return flux produced by the anti-solenoid coils passes between the two coils rather than fanning out and affecting the detector solenoid field. Design optimization is underway in order to provide the same anti-solenoid tuning knobs provided in an earlier brute force large coil design [4].

The two anti-solenoid coil support tubes are directly connected together. Initially it seemed natural to integrate the anti-solenoid coils with the $4.5 \mathrm{~K}$ heat shield surrounding the $1.9 \mathrm{~K}$ QD0 cold mass, but we soon realized that this had several negative implications for the heat shield (require better temperature stability for added complexity, increased cooling flow, more control valves, additional style of current lead needed). Instead we will integrate the anti-solenoid coils with the He-II cold mass.

\section{DETECTOR INTEGRATED DIPOLE}

The detector integrated dipole (DID) was originally invented to minimize orbit distortions and synchrotron radiation degradation to allow the ILC crossing angle to be increased to $25 \mathrm{mr}$ for an IP layout compatible with doing gamma-gamma collisions [5]. The DID adds a small horizontal field component to the detector field to more closely line the field up with the incoming beam. But putting a dipole at small radius deep inside an experiment is much too invasive. So we include a weak

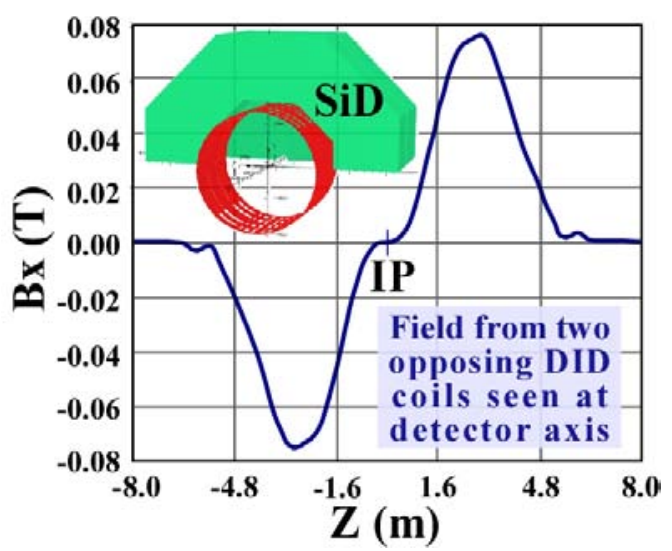

Figure 2. DID with bucking to reduce field near IP. Insert is SiD model with solenoid coil hidden to see DID coils. dipole winding in the detector solenoid cryostat where the coil is out of the way at large radius. The ILC crossing angle geometry implies that the DID field changes sign on either side of the IP and thus crosses zero right at the IP.

Subsequently there was intense interest in reducing the crossing angle to $14 \mathrm{mr}$ where a DID would not seem to be needed; however, reversing the DID field (sometimes referred to as an anti-DID) more closely aligns the detector field with the exiting beam instead. The anti-DID enables low energy particles to go out the exit beam pipe for reduced detector background. Still an issue surfaced for experiments that use time projection chamber (TPC) tracking and are sensitive to detector field perturbations. It was requested that the DID field be made small over some fiducial volume around the IP. Both the DID field and its slope should be small at the IP. In principle this can be achieved with multiple DID coils of different polarity that are longitudinally offset and buck one another.

The first result for a DID in this bucking configuration is shown in Fig. 2. The calculation is made for $\mathrm{SiD}$ and is fully three dimensional. We start from an approximate air coil, no yoke, solution and find that minor readjustment of DID coil currents takes care of differential saturation effects and we can easily zero both the field and its slope at the IP. The shape of the DID field distribution does however differ dramatically from that of an air coil in the region close to the detector end caps and detector specific calculations/optimizations must be done for each concept. While a first look at a DID engineering solution for SiD did not point out any show stoppers, many engineering details remain to be worked out which likely differ for each detector concept.

\section{TAIL FOLDING OCTUPOLES}

The remaining BDS superconducting magnets, the tailfolding octupoles, are more than $600 \mathrm{~m}$ away from the IP in an area devoid of cryogenic infrastructure (the end of the linac is even further away). Non-linear focusing of beam halo by pairs of octupole doublets reduces IP background and significantly relaxes tight BDS collimator jaw opening requirements [6].

We developed a novel superferric octupole design in order to reach the highest practical gradient while keeping production costs low. A high field octupole is especially challenging because an octupole's long narrow poles are 


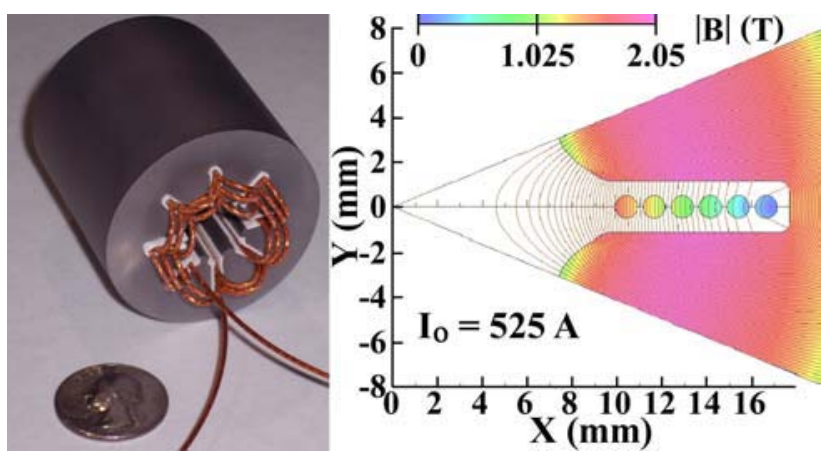

Figure 3. Tail-folding octupole winding concept and 2D $|\mathrm{B}|$ field intensity map plotted in the yoke and conductor.

easily saturated with relatively little magnetic flux making it to the pole tip unless the excitation current is close to the aperture. Our design concept is illustrated in Fig. 3.

The octupole yoke cross section is not subdivided into angular regions but is manufactured whole with all eight poles done together with slots for a single thickness of superconducting cable between each pole. The magnet section pictured in Fig. 3 was directly machined via EDM as specified in an Opera2d [7] output file from the two dimensional octupole model. For actual ILC production we intend to use punched yoke laminations; however, with only eight octupoles, each $2.5 \mathrm{~m}$ long, being needed, we expect to revisit the cost of punch tooling see if it remains cheaper than automatic EDM production.

The trick that makes this design viable, as opposed to assembling the yoke from individual sections with some type of micro-collar assembly to hold the magnet together, is that we use a variation of the Serpentine winding technique [8]. The octupole has an inner pole tip radius of $8 \mathrm{~mm}$ and the round seven strand $\mathrm{NbTi}$ superconducting cable diameter is just slightly more than $1 \mathrm{~mm}$. So it would be quite challenging to assemble a yoke around the coil in individual sections. Instead we push a single loop of superconducting cable through the entire $2.5 \mathrm{~m}$ long magnet and hook the loop over the end of the pole. Then we thermally plant the cable loop on either side of the pole in insulated slots. In this manner we work our way around the octupole cross section winding loops around alternate poles until we return to our starting point.

Here we have a choice of either continuing to wind around the octupole cross section with constant handedness or to reverse direction and wind loops around the pole ends we previously skipped. But winding with constant handedness gives a net solenoid winding around the beam tube (\# turns = \# layers). By winding an even number of layers and reversing the winding direction at each layer, we avoid making a net solenoidal field. A further benefit of such a procedure is that at each end the loops can be supported in individual end slots. The design shown in Fig. 3 has six layers with three turns per pole.

With our simplified production technique the largest remaining cost driver is the cryocooler needed for this cryogenically orphaned system. So we will insulate the individual strands of the round superconducting cable and use an external wiring patch to connect the strands in series. For a seven strand cable this reduces the operating current from $525 \mathrm{~A}$ to $75 \mathrm{~A}$ for significant cost savings thanks to using smaller capacity cryocoolers.

\section{SUMMARY AND FUTURE WORK}

For each detector concept we must still tackle difficult systems integration issues and MDI questions:

- How is the QD0 cryostat supported?

- How do we get helium, current leads and magnet instrumentation in/out?

- How are the above compatible with different detector access requirements?

- What sort of cryogenic umbilical connection allows the detector and QD0 cryostat systems to move cold about $20 \mathrm{~m}$ during a switchover?

- Given the above issues, what can we predict for QD0 operation (i.e. cool down, sensitivity to external perturbations and vibrations etc.)?

We are addressing many technical issues through ongoing $\mathrm{R} \& \mathrm{D}$ producing and testing a QD0 engineering prototype. Many important MDI issues will be taken up at a September 2007 ILC IR design workshop at SLAC.

We see that the ILC BDS superconducting magnets span an enormous range in design parameter space with apertures as small as $14 \mathrm{~mm}$ for the tail-folding octupoles (20 $\mathrm{mm}$ for QD0) to a $7 \mathrm{~m}$ coil diameter for the DID. QD0 has strong, $140 \mathrm{~T} / \mathrm{m}$, focusing for the incoming beam but its external field is small just a few millimeters beyond the coil outer surface. The force neutral antisolenoid is dramatically superior compared to the original brute force concept. But much work still remains on complex MDI and push-pull issues to find workable, cost effective, systems integrated solutions for the machine, the detector and the BDS superconducting magnets.

\section{REFERENCES}

[1] P. Wanderer, et. al., "Completion of Superconducting Magnet Production by BNL for HERA Luminosity Upgrade," MT-17, Geneva, Switzerland, (2001).

[2] B. Parker, "Recent Progress Designing Compact Superconducting Final Focus Magnets for the ILC," Nanobeam05, Kyoto, Japan (2005).

[3] A. Seryi, et. al., "Design of the Beam Delivery System for the International Linear Collider," paper WEOCAB01, this conference.

[4] Y. Nosochkov and A. Seryi, "Compensation of detector solenoid effects on the beam size in a linear collider," Phys. Rev. ST Accel. Beams, 8, 021001, (2005).

[5] B. Parker and A. Seryi, "Compensation of effects of a detector solenoid on the vertical beam orbit in a linear collider," Phys. Rev. ST Accel. Beams 8, 041001 (2005).

[6] R. Brinkmann, et. al., "Halo reduction by means of nonlinear optical elements in the NLC final focus system," SLAC-PUB-8896, PAC01, (2001).

[7] Opera2d code, Vector Fields Ltd, Oxford, UK.

[8] B. Parker and J. Escallier, "Serpentine Coil Topology for BNL Direct Wind Superconducting Magnets," PAC05, (2005). 